\title{
A New Trajectory Optimizing Method Using Input Shaping Principles
}

\author{
Pu Zhao, ${ }^{1}$ Yunfei Zhou, ${ }^{1}$ and Rougang Zhou $\mathbb{D}^{2,3}$ \\ ${ }^{1}$ State Key Lab of Digital Manufacturing Equipment and Technology, Huazhong University of Science and Technology, \\ 1037 Luoyu Road, Wuhan 430074, China \\ ${ }^{2}$ School of Mechanical Engineering, Hangzhou Dianzi University, 1158 Baiyang Road, Hangzhou 310000, China \\ ${ }^{3}$ Mstar Technologies, Inc, \#3 Xidoumen Road, Hangzhou 310000, China \\ Correspondence should be addressed to Rougang Zhou; zhourg@hdu.edu.cn
}

Received 24 June 2018; Accepted 13 August 2018; Published 18 September 2018

Academic Editor: Jean-Mathieu Mencik

Copyright (c) $2018 \mathrm{Pu}$ Zhao et al. This is an open access article distributed under the Creative Commons Attribution License, which permits unrestricted use, distribution, and reproduction in any medium, provided the original work is properly cited.

\begin{abstract}
Input shaping is an efficient control technique which has motivated a great number of contributions in recent years. Such a technique generates command signals that can manoeuvre flexible structures without exciting their vibration modes. This paper introduces a new trajectory optimizing method based on input-shaping principles. The main characteristic of this method in comparison with traditional input-shaping technique is the generating process of final trajectory. By adding reversion and postprocessing procedures before input shapers, time-delay and most of the trajectory deviation can be removed. The improvement of the proposed method compared with a traditional method is evaluated by simulations. It will be shown that the proposed method leads to no time-delay and highly decreased trajectory deviation and little change of robustness.
\end{abstract}

\section{Introduction}

Many mechanical systems are characterized by parasitic vibrations, such as flexible robots, slewing flexible spacecraft, cranes, etc [1]. In recent years, there has been much effort on optimizing trajectories which lead to reduced residual vibration or overshoot. Various well-designed motion profiles, such as trapezoidal, S-curve, trigonometric functions are well-known methods for smooth motion control $[2,3]$. By using the response pattern of specific functions, reduced residual vibration trajectory can be obtained. However, these methods are based on parameter selection of specific motion equation. When other kinds of trajectories are required, these methods are unsuitable [4]. This inherent drawback highly restricts the use of well-designed profiles.

Next to well-designed motion profiles, input-shaping technique (IST) is another well-known technique through which to generate signals that do not excite the resonance modes, whilst the final position is attained without steadystate errors as shown in Figure 1(a) [5]. By convolving the reference input signal with a series of impulses, the vibratory mode excited by the signal can be effectively cancelled [6] Thanks to its advantages, such as simplicity, effectiveness, and extensive applicability, IST has received much attention in the control theory and led to many engineering applications [7]. Zero vibration $(\mathrm{ZV})$ shapers, zero vibration derivative (ZVD) shapers and zero vibration derivative-derivative (ZVDD) shapers were first developed [8]. Then, more robust shapers, e.g., extra insensitive (EI) shaper, multihump input shaper and perturbation-based extra insensitive (PEI) input shaper, were developed by researchers $[7,9,10]$. However, when using these shapers, there are two key trade-offs: shaper length and parameter robustness [11]. To solve these problems, learning input shapers and adaptive input shapers were developed by researchers $[12,13]$. These shaping methods are designed by using a short input shaper that is less robust and change its parameters in tune with the system. Besides, input shapers were also developed to deal with time-varying and multimode cases. However, these improvements mainly focus on the shapers' robustness or parameter selection, and little research has been paid on the drawbacks caused by IST itself. As the input shapers are decided, trajectory deviation and time-delay will be caused 


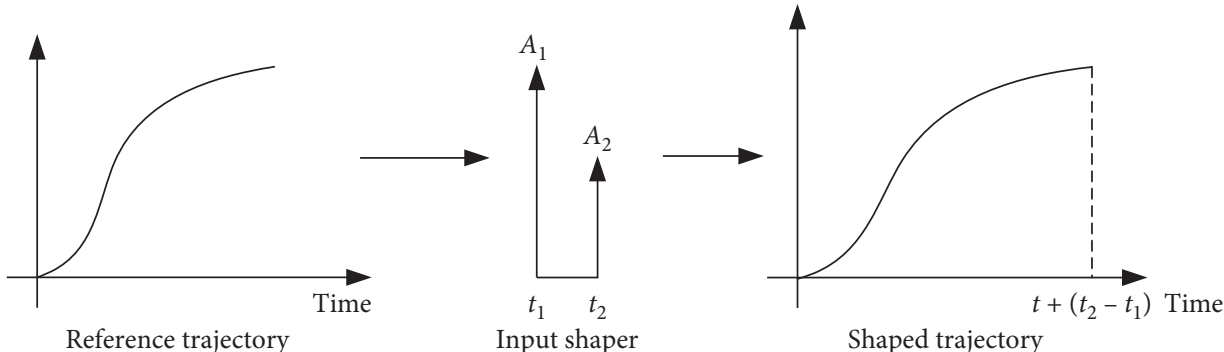

(a)

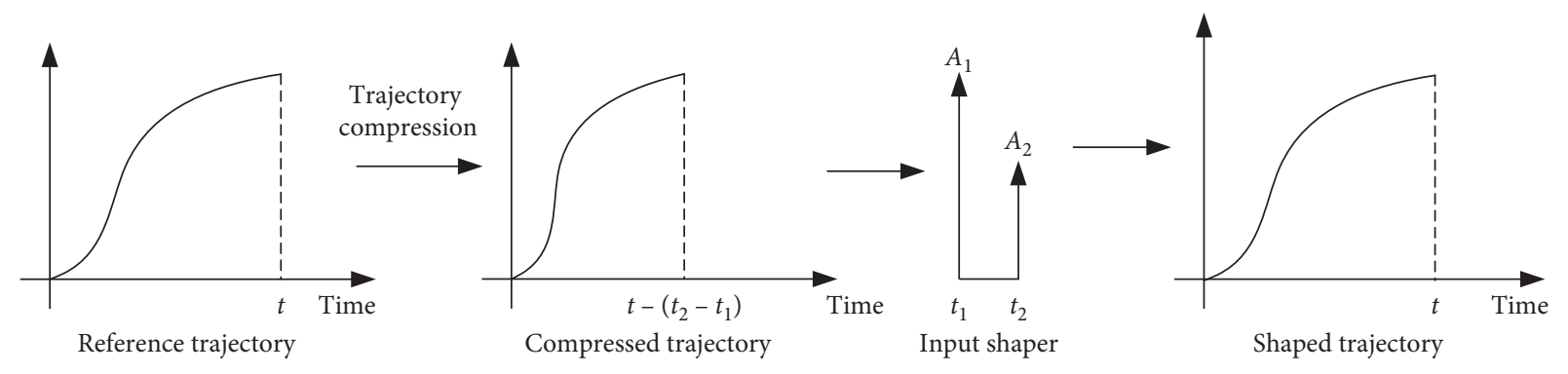

(b)

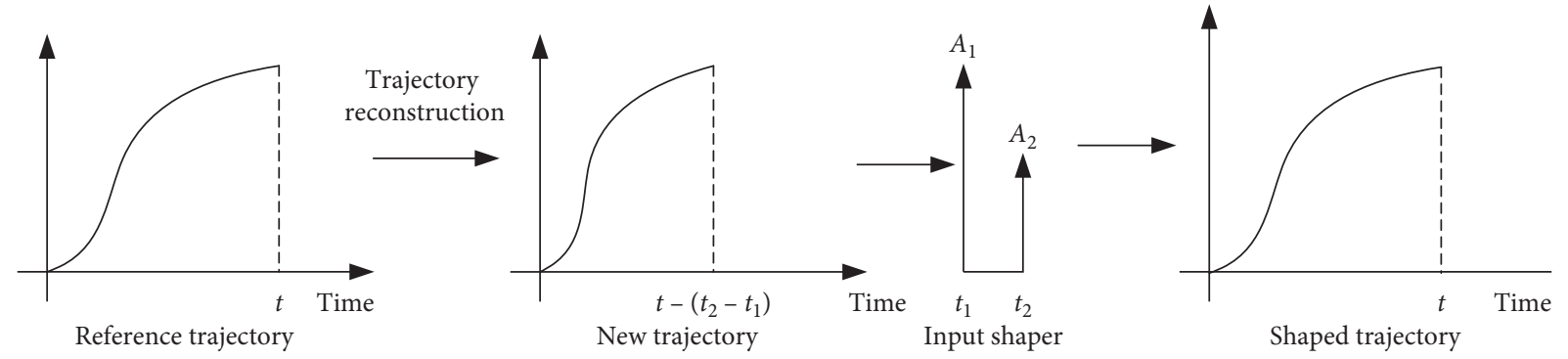

(c)

FIgure 1: Principles of several IST: (a) traditional IST, (b) C-IST, and (c) proposed R-IST.

in convolving process, as shown in Figure 1(a). By adding trajectory compression process (C-IST), time-delay can be removed, as shown in Figure 1(b) [14]. However, damage of shaper's robustness will happen and trajectory deviation still remains. It should be noted that target trajectories are always designed based on different criteria $[15,16]$. As a result, less trajectory deviation and less change of robustness will be preferred. To our best knowledge, there is no such a report concentrating on this target.

In this report, a new trajectory optimizing method using IST principles is proposed to solve the two drawbacks to some extent. The basic idea of this method is shown in Figure 1(c). Through an additional trajectory reconstruction process, a new trajectory is obtained before using IST. The reconstruction process is carried out with the decomposition operation of target trajectory based on IST principles. Spectrum analysis and postprocessing of the obtained trajectory is then carried out. Finally, inputshaping process is implemented. So, the method can be called reversion-input-shaping technique (R-IST). As verified by simulation results, the proposed R-IST is able to remove time-delay phenomenon, reduce a large proportion of trajectory deviation, and change little robustness of the shapers. Since the revision process includes some complex calculation, this method is suitable to be used in off-line trajectory optimizing process.

This report is organized as follows. In Section 2, the traditional IST is revisited. The main steps of R-IST are demonstrated using an example in Section 3. In Section 4, simulation results and discussions are carried out about the new method. Finally, in Section 5, the conclusion follows.

\section{Preliminaries}

An uncoupled, linear, vibratory system of any order can be specified as a cascaded set of second-order systems [8]. So, consider a second-order linear system:

$$
\ddot{y}(t)+2 \xi \omega_{0} \dot{y}(t)+\omega_{0}^{2} y(t)=\omega_{0}^{2} u(t),
$$

where $\omega_{0}$ and $\xi$ are the natural frequency and the damping ratio, respectively; $u$ and $y$ means input and output of the system. Then impulse response $w(t)$ of the system can be expressed as 


$$
\begin{aligned}
w(t) & =L^{-1}\left[\frac{\omega_{0}^{2}}{s^{2}+2 \xi \omega_{0} s+\omega_{0}^{2}}\right] \\
& =\frac{\omega_{0}}{\sqrt{1-\xi^{2}}} e^{-\xi \omega_{0} t} \sin \left(\omega_{0} \sqrt{1-\xi^{2}}\right) t,
\end{aligned}
$$

where $L^{-1}(\cdot)$ means the inverse Laplace transformation. The input-shaping method convolves the original command with a sequence of impulses, as shown in Figure 1. To generalize trajectory without residual vibration, amplitudes and moments of applied impulses need to be precisely selected. The input shaper may be expressed in the time domain as

$$
f(t)=\sum_{i=1}^{n} A_{i} \delta\left(t-t_{i}\right)
$$

where $A_{i}$ is the amplitudes of the $i$ th impulse, $t_{i}$ is the applied time moments of the $i$ th impulse, and $t_{n}$ is the time at which the sequence ends (time of the last impulse). Function $\delta$ means unit-impulse function. The response to the impulses of the input shaper can be expressed by

$$
y(t)=f(t) * w(t)=A \sin \left(\omega_{0} \sqrt{1-\xi^{2}} t+\varphi\right),
$$

where

$$
\begin{aligned}
& A=\sqrt{\left(\sum_{i=1}^{n} B_{i} \sin \phi_{i}\right)^{2}+\left(\sum_{i=1}^{n} B_{i} \cos \phi_{i}\right)^{2}}, \\
& \varphi=\arctan \left[\frac{\left(\sum_{i=1}^{n} B_{i} \sin \phi_{i}\right)}{\left(\sum_{i=1}^{n} B_{i} \cos \phi_{i}\right)}\right] \\
& \phi_{i}=\omega_{0} \sqrt{1-\xi^{2}} t_{i} \\
& B_{i}=\frac{A_{i}}{\sqrt{1-\xi^{2}}} e^{-\xi \omega_{0}\left(t-t_{i}\right)}
\end{aligned}
$$

In order to obtain zero vibration residual result when $t>t_{n}, A_{i}$, and $t_{i}$ to be determined should satisfy

$$
\begin{aligned}
& \sum_{i=1}^{n} A_{i} \omega_{0} e^{-\xi \omega_{0}\left(t_{n}-t_{i}\right)} \cos \left(\omega_{0} \sqrt{1-\xi^{2}} t_{i}\right)=0, \\
& \sum_{i=1}^{n} A_{i} \omega_{0} e^{-\xi \omega_{0}\left(t_{n}-t_{i}\right)} \sin \left(\omega_{0} \sqrt{1-\xi^{2}} t_{i}\right)=0 .
\end{aligned}
$$

In addition, another two constraints should also be satisfied, as shown by

$$
\begin{aligned}
\sum_{i=1}^{n} A_{i} & =1, \\
t_{1} & =0 .
\end{aligned}
$$

These two constraints reflect the choice of time origin and the requirement that input shapers do not change the amplitude of trajectory. From the above equations, the parameters to be designed still can't be determined. Shan and other researchers have given various methods by adding different constraints [17]. In this place, Singer's solution was selected, as shown by

$$
\begin{aligned}
A_{i} & =\frac{C_{n-1}^{i-1} K_{i-1}}{\sum_{i=1}^{n} C_{n-1}^{i-1} K_{i-1}}, \\
t_{i} & =\frac{(i-1) \pi}{\omega_{n} \sqrt{1-\xi^{2}}}, \\
K_{i-1} & =e^{-(i-1) \xi \pi / \sqrt{1-\xi^{2}}}, \\
C_{n-1}^{i-1} & =\frac{(n-1) !}{(i-1) !(n-i) !} .
\end{aligned}
$$

Zero vibration $(\mathrm{ZV})$ shapers and zero vibration derivative (ZVD) shapers can be calculated by selecting parameter $n$ to be 2 and 3, respectively.

\section{Main Principle of R-IST}

The R-IST to be presented involves some extra necessary steps, which mainly concentrate on removing spikes or highfrequency components of applied trajectory. In order to demonstrate R-IST and additional steps that need to be used, a specific application was taken as an example. Figure 2 shows a simple mass-spring-damper system. This System can be seen as a second-order linear system.

Dynamical model of the system can be expressed by (9). Then, the parameters of the input shaper can be decided by (1) and (8):

$$
m \ddot{y}(t)+c \dot{y}(t)+k y(t)=-m \ddot{x}(t),
$$

where $m$ denotes the mass of the load and $k$ and $c$ present the stiffness and damping of the spring. During the simulation process, $m$ is selected as $2 \mathrm{~kg}$, while $k$ and $c$ are selected as $200 \mathrm{~N} / \mathrm{m}$ and $0.05 \mathrm{Ns} / \mathrm{m}$, respectively. Since this research is focused on decrease trajectory deviation caused by IST, comparison between R-IST and traditional IST using the same shapers are selected. In the following deduction of R-IST, it can be shown that different shapers are able to be applied in R-IST. Since some properties can be obtained by reversion process using $\mathrm{ZV}, \mathrm{ZVD}$, and $\mathrm{ZVDD}$ shapers, these shapers are selected. Because ZVD shaper has better robustness than ZV shaper and less time-delay than ZVDD shaper, ZVD shaper was selected to compare trajectory deviation, robustness, and time-delay properties between R-IST and IST. Since the mentioned methods are able to use different kinds of input shapers, we use R-ZVD to express R-IST based on ZVD shaper. Parameters of ZVD shaper can be selected by (8). During the derivation process, a trapezoidal velocity motion profile is selected as target trajectory $\mathbf{S}_{0}$, as shown in Figure 3.

The main principle of reversion process is to calculate a new trajectory, called the origin trajectory, which is similar with target trajectory after processed by input shapers. Sorensen and his colleagues have proposed a calculation method based on deconvolution process [18]. However, this 


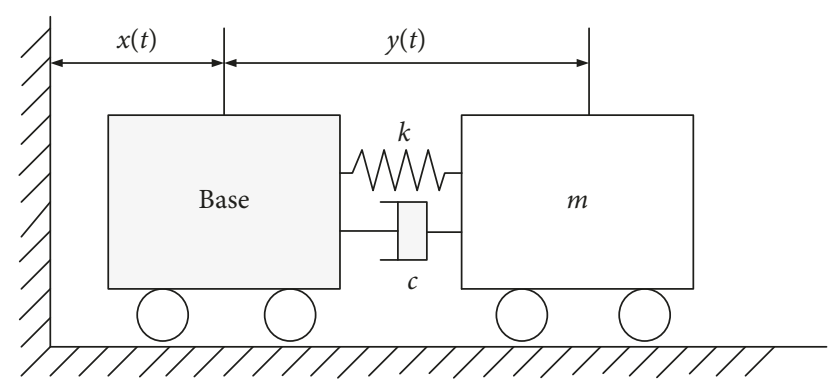

Figure 2: Modeling of a flexible system.
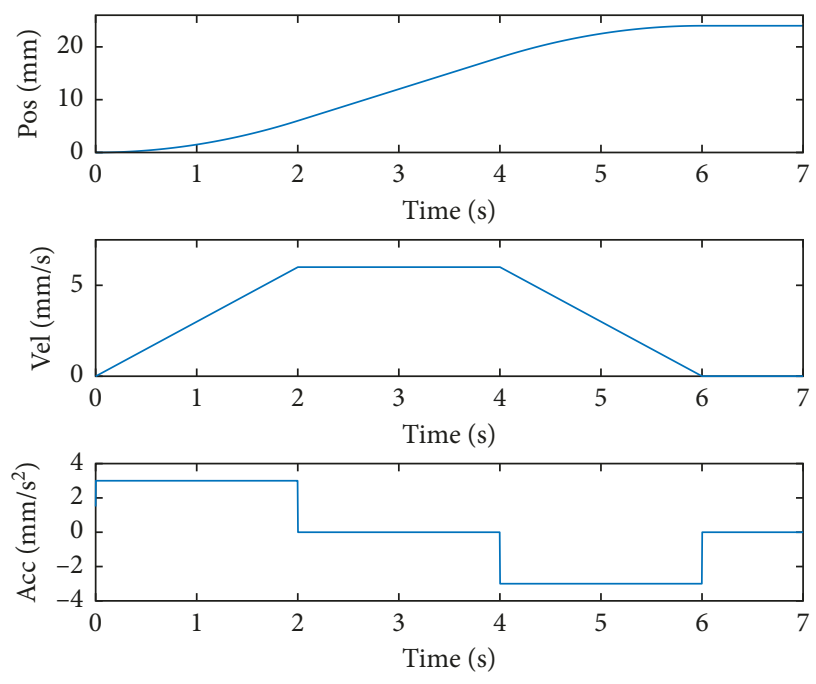

FIgURE 3: Motion profile of target trajectory.

method will cause inevitable spikes in acceleration map of obtained trajectory and will lead to overload of actuators. In this paper, a discrete calculating method based on matrix manipulation is selected. At first, the trajectory is discretized so that matrix operation can be carried out. The interval between two discrete points is selected as $2 \mathrm{~ms}$. Trajectory $\mathbf{S}_{0}$ becomes a column vector, where each element means displacement in corresponding moment. Also, input shapers should also be discretized. Because the time moment of shaper's impulse may not be integer multiples of the time interval, the interpolation method should be introduced. In our application, the linear interpolation method is selected for its simplicity when expressed by matrix. Velocity and acceleration coefficient matrix, which are used to calculate velocity and acceleration profiles, can be easily obtained by using finite difference method, as shown by

$$
\begin{aligned}
& v(t)=\frac{S(t+\Delta t)-\mathrm{S}(t)}{\Delta t}, \\
& a(t)=\frac{\mathrm{S}(t+\Delta t)-2 \mathrm{~S}(t)+\mathrm{S}(t-\Delta t)}{\Delta t^{2}},
\end{aligned}
$$

where $a$ and $v$ means acceleration and velocity in $t$ moment, respectively, and $\Delta t$ expresses time interval of discretization process. After the discretization process, the origin trajectory can be calculated using matrix operation, as shown by:

$$
\left\{\mathbf{S}_{0}\right\}=\left[\mathrm{COE}_{1}\right]\left\{\mathbf{S}_{1}\right\}+\left\{\mathbf{e}_{1}\right\},
$$

where $S_{1}$ and $\mathbf{e}_{1}$ denote the origin trajectory to be calculated and residual errors, respectively. Besides, matrix $\mathbf{C O E}_{1}$ is the input-shaping calculation matrix and has the same effect with $f(t)$ in (4). Each row of $\mathbf{C O E}_{1}$ represents the applied input shapers on corresponding moment and can be easily formed by (3). It should be noted that for any trajectory $\mathbf{S}_{0}$ with moving time longer than the selected shaper, matrix $\mathbf{C O E}_{1}$ is always full rank. As a result, (11) is an overdetermined equation when $\mathbf{e}_{1}$ is selected as zero. So the equation can be solved by least minimum square (LMS) method.

Obtained trajectory $\mathbf{C O E}_{1} * \mathbf{S}_{1}$ by (11) is expressed by Figure 4(a). However, this result cannot be used directly. As can be seen in the picture, the acceleration profile result is characterized by high-frequency components and large amplitude. This phenomenon is caused by LMS and will be discussed in detail in Section 4.3. To solve the problem, the weighted least minimum square (W-LMS) method is introduced. Solving equation is changed from (11) to (12):

$$
\left\{\begin{array}{c}
\mathbf{S}_{0} \\
k_{1} \mathbf{S}_{a}
\end{array}\right\}=\left[\begin{array}{c}
\mathrm{COE}_{1} \\
k_{1} \mathbf{C O E}_{a}
\end{array}\right]\left\{\mathbf{S}_{1}\right\}+\left\{\begin{array}{c}
\mathbf{e}_{1} \\
k_{1} \mathbf{e}_{2}
\end{array}\right\},
$$

where $\mathbf{S}_{a}$ and $\mathbf{C O E}_{a}$ means the target acceleration profile and acceleration calculation matrix. Besides, $k_{1}$ and $e_{2}$ means weight factor and residual error. Parameter $k_{1}$ is used to balance the trade-offs: residual deviation and high-frequency components. Compared with the optimization method, LMS-like method costs much less computation time because there is no need for iteration.

As resonance part will highly damage the robustness of target trajectory, it is reasonable to filter out the resonant components. Through comparing spectrum of $\mathbf{S}_{0}$ and $\mathbf{S}_{1}$, differences between 0 and $6 \omega_{0}$ are filtered out as $S_{\text {filtered }}$. In the filtering process, upper limit of the bandwidth is selected as $6 \omega_{0}$ because higher frequency components have little effect to the second-order system (9). When faced with multidegree of freedom systems, the maximum of natural frequency should be selected as $\omega_{0}$. After using the filter, acceleration profile in frequency domain is changed from Figures 5(a) and 5(b). Then trajectory $\boldsymbol{S}_{1}$ can be expressed as

$$
\left\{\mathbf{S}_{1}\right\}=\left\{\mathbf{S}_{\text {pass }}\right\}+\left\{\mathbf{S}_{\text {filtered }}\right\},
$$

where $\boldsymbol{S}_{\text {filtered }}$ denotes the filtered parts in this step, while $\mathbf{S}_{\text {pass }}$ denotes the remaining components. However, the filtering operation is accomplished in acceleration profiles, which will lead to extra alteration of trajectory. As shown by Figure $6, \mathbf{S}_{\text {filtered }}$ has low frequency components and resonance components. Resonance components will cause damage of shaper robustness in obtained new trajectory and should be removed. Only the resonance components need to be wiped out, while the low frequency components to be kept, as shown by 

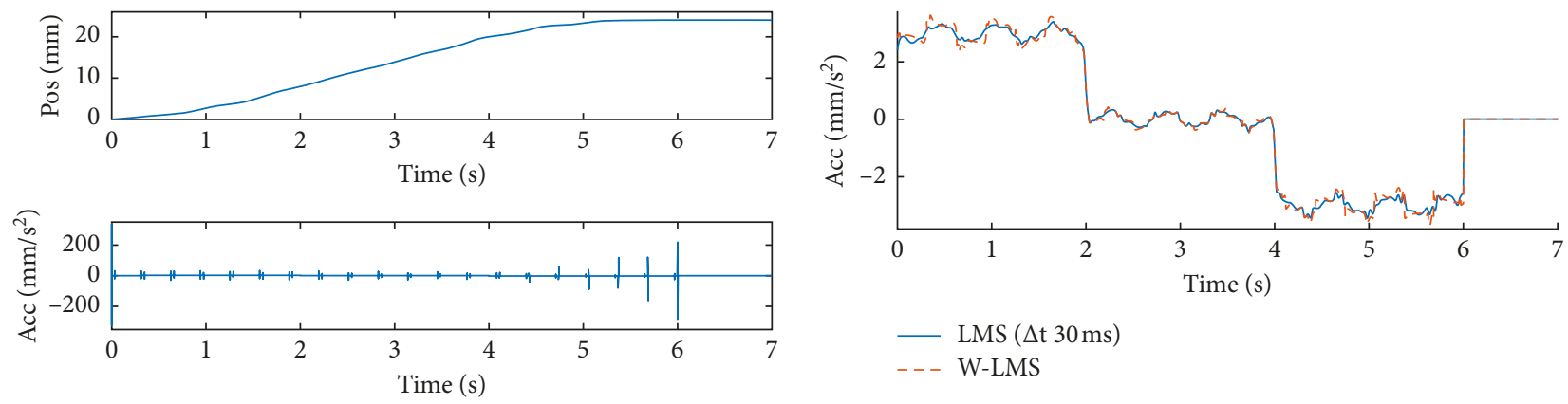

$-\operatorname{LMS}(\Delta \mathrm{t} 30 \mathrm{~ms})$

-.- W-LMS
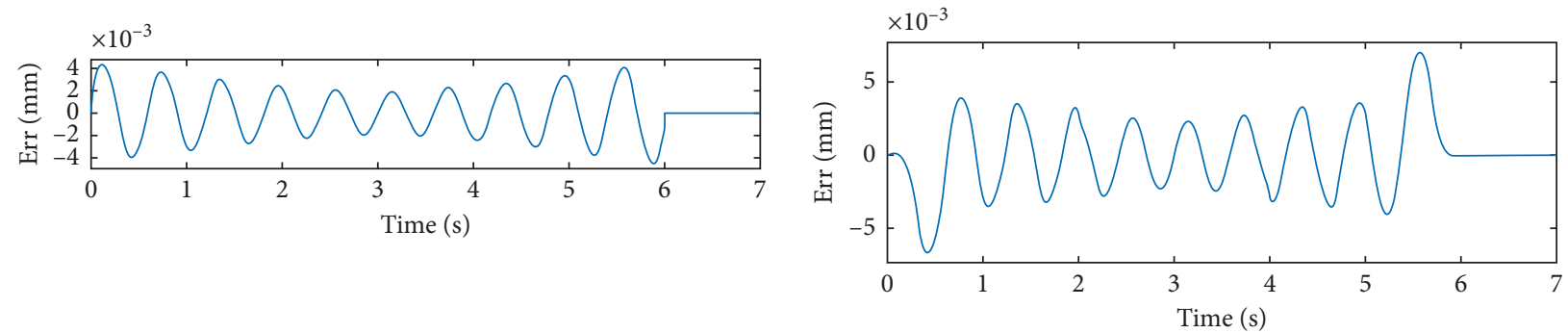

(a)

(b)

FIGURE 4: Reversion results obtained by LMS and W-LMS: (a) obtained motion profile of target trajectory solved by LMS and (b) acceleration profile of target trajectory solved by W-LMS.

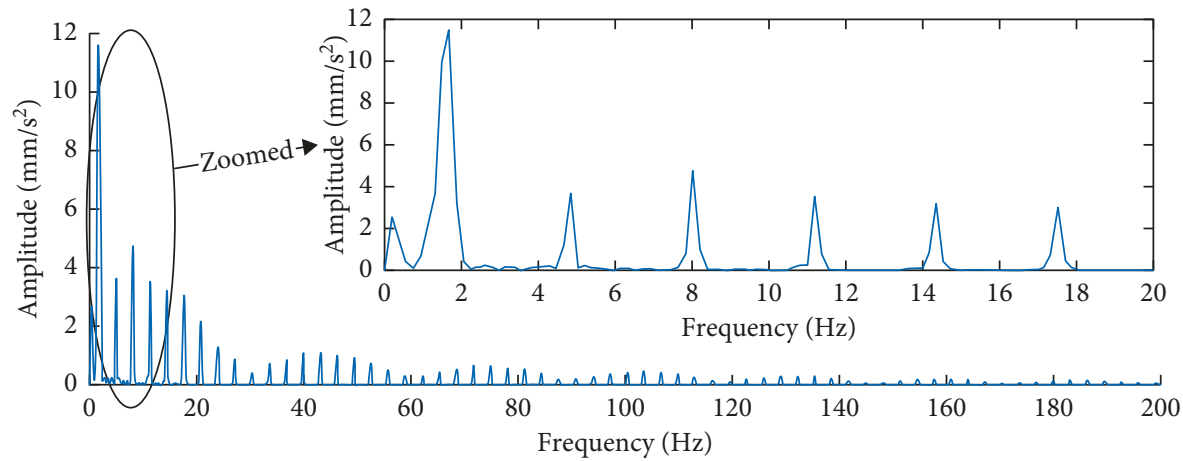

(a)

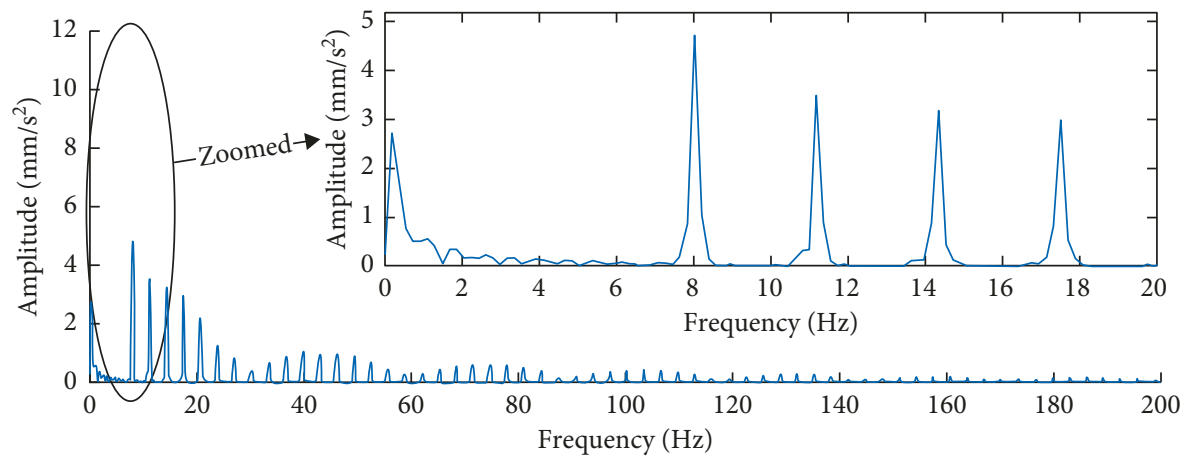

(b)

Figure 5: Spectrum of acceleration profile of $\boldsymbol{S}_{1}$ using filter and filtered acceleration profile: (a) acceleration profile of $\mathbf{S}_{1}$ and (b) filtered acceleration profile. 


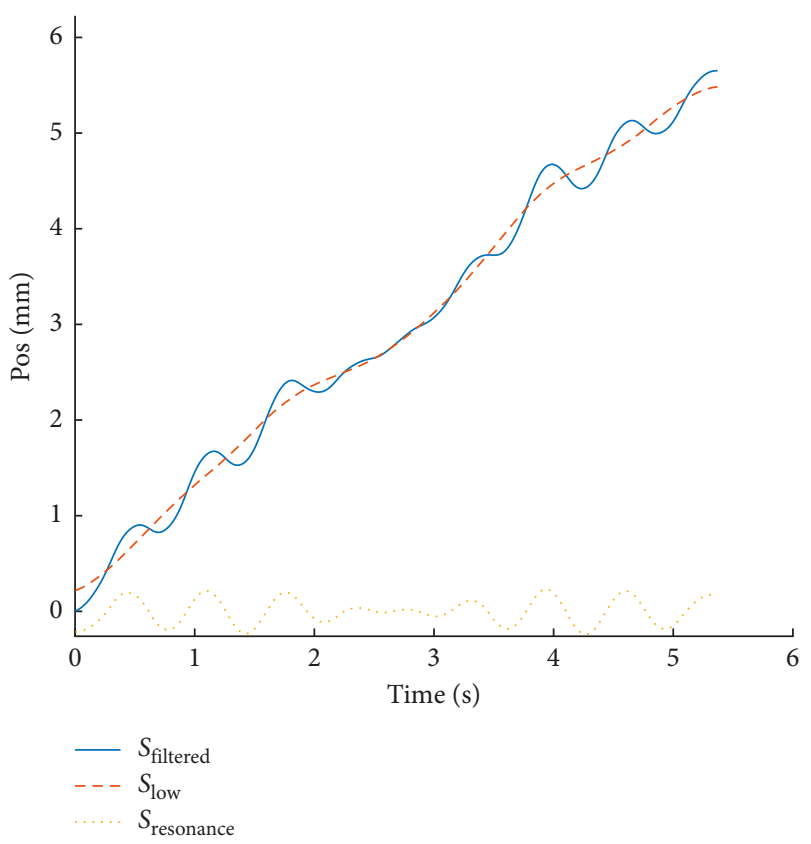

Figure 6: Filtered trajectory decomposition using EMD.

$$
\begin{aligned}
\left\{\mathbf{S}_{1}\right\} & =\left\{\mathbf{S}_{\text {pass }}\right\}+\left\{\mathbf{S}_{\text {filtered }}\right\} \\
& =\left\{\mathbf{S}_{\text {pass }}\right\}+\left\{\mathbf{S}_{\text {low }}\right\}+\left\{\mathbf{S}_{\text {resonance }}\right\} \\
& =\left\{\mathbf{S}_{2}^{\prime}\right\}+\left\{\mathbf{S}_{\text {resonance }}\right\},
\end{aligned}
$$

where $\mathbf{S}_{\text {resonance }}$ denotes the resonance components.

Empirical mode decomposition (EMD) has been applied widely in signal processing field. This method has the ability to separate period signal from complex signal sources [19]. This method was selected because $\mathbf{S}_{\text {filtered }}$ can appear as such a signal source. By using EMD, $\mathbf{S}_{\text {filtered }}$ is decomposed into two parts, as shown by Figure 6 and (14). It should be noticed that the starting point and final point of $\mathbf{S}_{2}^{\prime}$ is different with $S_{1}$. As a result, further compensation need to be introduced. Another trajectory $\mathbf{S}_{\text {2add }}$, which is similar with $\mathbf{S}_{2}^{\prime}$, is added. After the compensating process, (14) becomes (15):

$$
\begin{aligned}
\left\{\mathbf{S}_{1}\right\} & =\left\{\mathbf{S}_{2}^{\prime}\right\}+\left\{\mathbf{S}_{\text {resonance }}\right\}+\left\{\mathbf{S}_{\text {2add }}\right\}-\left\{\mathbf{S}_{\text {2add }}\right\} \\
& =\left\{\mathbf{S}_{2}\right\}+\left\{\mathbf{S}_{\text {resonance }}\right\}-\left\{\mathbf{S}_{\text {2add }}\right\}=\left\{\mathbf{S}_{2}\right\}+\left\{\mathbf{e}_{2}^{\prime}\right\},
\end{aligned}
$$

where $\mathbf{e}_{2}^{\prime}$ means deviation generated during this process.

As trajectory $S_{2}$ had already been obtained, (11) could be used to calculate final trajectory $\mathbf{S}_{0}^{\prime}$. Acceleration profile of trajectory $S_{0}^{\prime}$ is shown by Figure $7(\mathrm{a})$. It should be noticed that $\mathbf{S}_{0}^{\prime}$ still can't be used directly because of the spikes. As described before, W-LMS method was selected again to remove these spikes. So, target acceleration profile should be obtained at first. Different with former large amplitudes generated in reversion process, the spikes can be removed by signal processing methods. Wavelet transformation has been used widely for filtering out local spikes in the area of signal processing [20]. In our simulation, Haar wavelet is selected because of its simplicity. As shown in Figure $7(\mathrm{~b})$, the acceleration profile is decomposed in 6 levels. In the picture, $a_{i}$ means the approximations at level $i$, while $d_{i}(i=2, \ldots, 6)$ means detail part of the profile in level $i$. By using this method, the local spikes in $\mathbf{S}_{0}^{\prime}$ can be separated and removed. Then, the weighted function can be expressed by

$$
\left\{\begin{array}{c}
\mathbf{S}_{2} \\
k_{2} a_{6}
\end{array}\right\}=\left[\begin{array}{c}
\mathbf{E} \\
k_{2} \mathbf{C O E}_{a}
\end{array}\right]\left\{\mathbf{S}_{3}\right\}+\left\{\begin{array}{c}
\mathbf{e}_{3}^{\prime} \\
\mathbf{e}_{4}
\end{array}\right\},
$$

where $k_{2}$ means weighting parameter to be selected properly. In our simulation, $k_{2}$ is chosen to be $0.01 . \mathbf{e}_{4}$ and $\mathbf{e}_{3}^{\prime}$ represent residual deviation, and $S_{3}$ denotes the obtained trajectory in this step. This equation is also an overdetermined equation.

Finally, through (11)-(16), the R-IST can be expressed by

$$
\begin{aligned}
\left\{\mathbf{S}_{0}\right\} & =\left[\mathrm{COE}_{1}\right]\left\{\mathbf{S}_{3}\right\}+\left[\mathrm{COE}_{1}\right]\left\{\mathbf{e}_{1}^{\prime}+\mathbf{e}_{2}^{\prime}+\mathbf{e}_{3}^{\prime}\right\}, \\
& =\mathbf{S}_{\mathrm{f}}+\left\{\mathbf{e}_{1}+\mathbf{e}_{2}+\mathbf{e}_{3}\right\}=\mathbf{S}_{\mathrm{f}}+\{\mathbf{e}\},
\end{aligned}
$$

where $\mathbf{e}_{1}, \mathbf{e}_{2}$, and $\mathbf{e}_{3}$ are trajectory deviation introduced in each step. $\mathbf{S}_{\mathrm{f}}$ and $\mathbf{e}$ denote the final obtained original trajectory and total trajectory deviation generated by R-IST, respectively. Acceleration profiles of trajectory $\mathbf{S}_{\mathrm{f}}$ in time domain and frequency domain are shown in Figure 8(a). Besides, $\mathbf{e}_{1}, \mathbf{e}_{2}, \mathbf{e}_{3}$, and $\mathbf{e}$ are also shown in Figure 8(b). As shown in Figure 8(b), $\mathbf{e}_{2}$ occupied the major part of the total trajectory deviation, which means the deviation is mainly caused by removing the resonance components using EMD. As a result, it is easy to estimate the generated trajectory deviation after using EMD.

\section{Simulation Results and Discussion}

To simulate the properties of the generated trajectory, Simulink was selected as simulation software.

4.1. Residual Vibration and Trajectory Deviation. Except the mentioned methods, trajectory compression before using input shapers, called C-IST, is also able to remove timedelay. In the following discussion, we use C-ZVD to express C-IST based on ZVD shaper. Responses to trajectory obtained by ZVD, R-ZVD, and C-ZVD are shown in Figure 9. To be compared, response to target trajectory is also demonstrated. It can be seen that target trajectory generates residual oscillation, while ZVD method and R-IST method lead to zero vibration residual. Besides, ZVD method generates the least vibration during the movement. It should be noted that there exists three platforms using ZVD and C-ZVD. It is because in these regions, the system is balanced and the vibration caused by 3 pulses was cancelled. As for the results caused by R-ZVD method, larger vibration was caused because the acceleration is no longer constant which could excite the resonance of the system. However, the vibration curve had the similar trend with ZVD method. It is because a new reversion process is added to decrease the deviation of the target trajectory before using ZVD shapers while the selected ZVD shapers are the same. Those changes alerted the balance state of ZVD method, which leads to no platform region. Besides, it should be noted that ZVD 


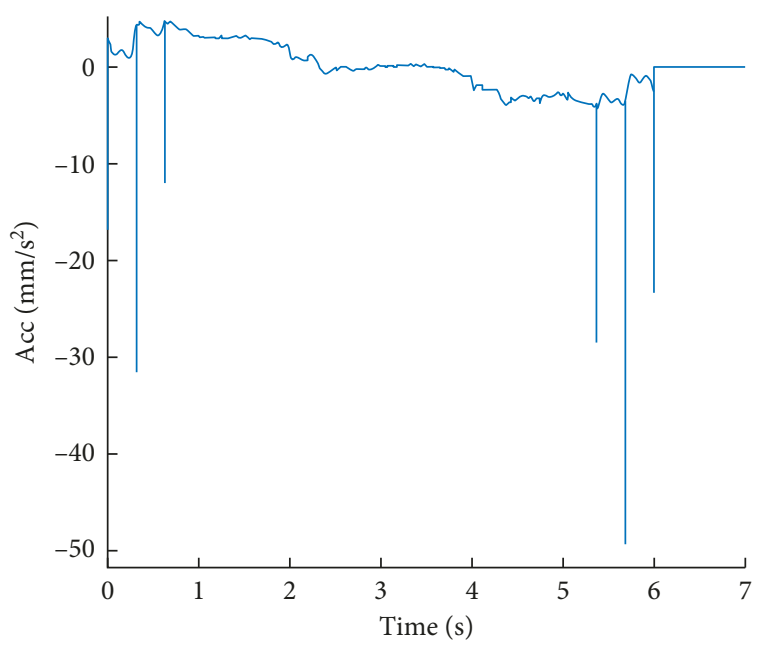

(a)
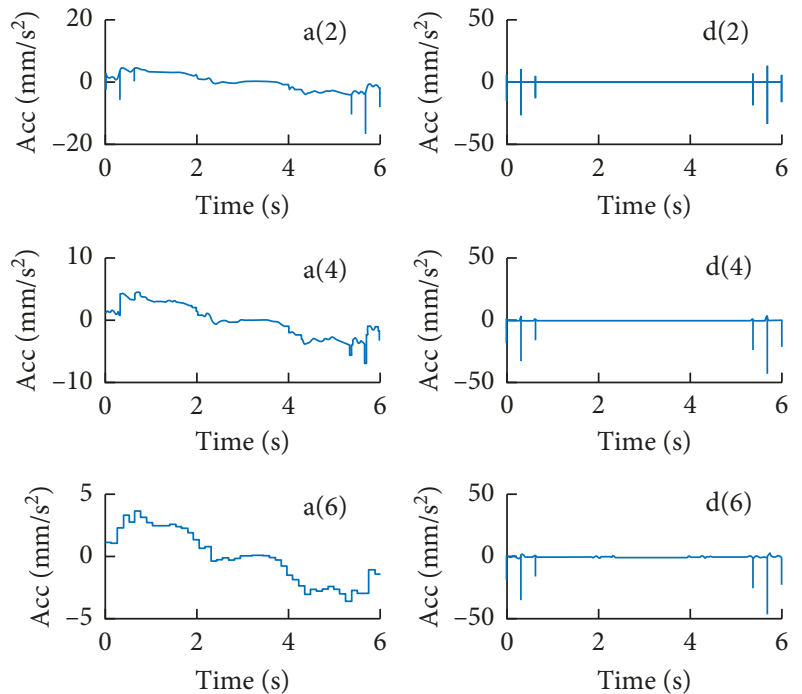

(b)

Figure 7: Acceleration profile of $\mathbf{S}_{0}^{\prime}$ and its wavelet decomposition: (a) acceleration profile of $\mathbf{S}_{0}^{\prime}$ and (b) wavelet decomposition of acceleration profile of $\mathbf{S}_{0}^{\prime}$ in 2,4 , and 6 levels.

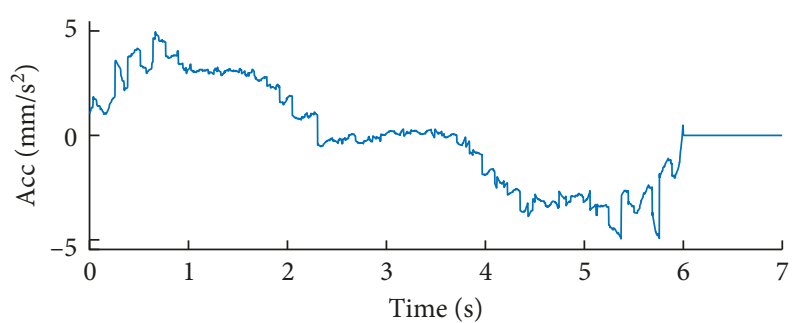

(a)

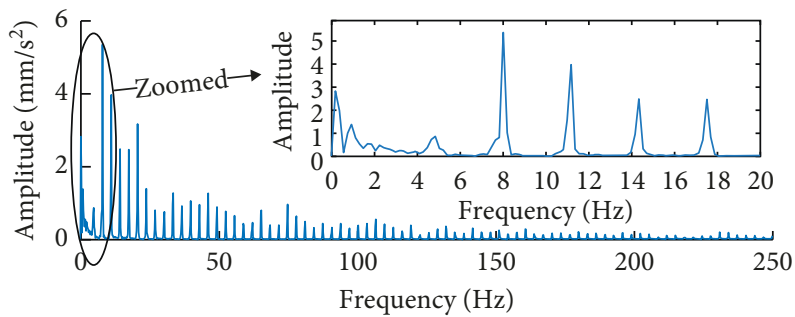

(b)

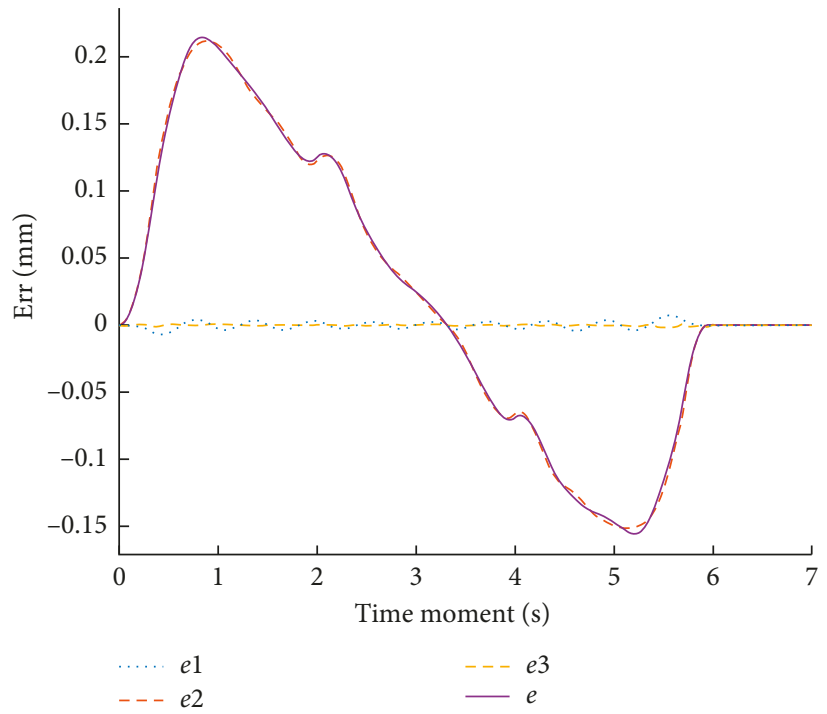

(c)

FiguRE 8: Final trajectory $\mathbf{S}_{\mathrm{f}}$ obtained by R-ZVD and generated errors in each step: (a) acceleration profiles, (b) spectrum of acceleration profiles, and (c) residual errors generated in each step. 


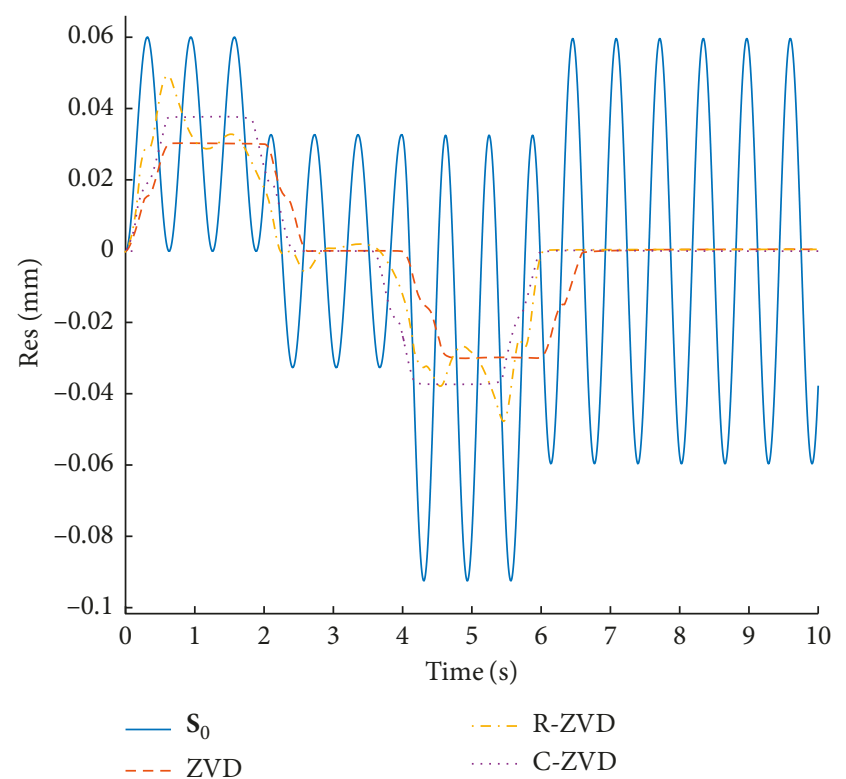

FIGURE 9: System responses to trajectory generated by different methods.

method generated time-delay while R-ZVD and C-ZVD did not.

Deviation of the target trajectory when using these methods is also simulated, as shown in Figure 10. Compared with generated vibration, trajectory deviation amplitude is much larger. It should be noticed that ZVD method leads to larger deviation, while R-IST the smaller. Deviation caused by ZVD method is always unidirectional, because the deviation is caused by time-delay. However, by using reversion process, R-IST generates less deviation. It is because the R-IST method is concentrated on generating trajectory with the smallest deviation and least resonant excitation source. Besides, trajectory deviation of R-ZVD is mainly caused by filtering the resonance part, as shown in Figure 8(c). Also, the deviation of C-ZCD is also decreased when compared with ZVD. The reason is that C-ZVD change the deviation from unidirectional to anisotropic, which alleviate the influence of time-delay. By Figures 9 and 10, it can be seen that the R-ZVD method generates the least trajectory deviation. Compared with traditional ZVD, $88.6 \%$ less deviation in root mean square (RMS) was obtained, which shows the priorities of this method in reducing trajectory deviation.

4.2. Robustness Properties. Except those applied in closeloop systems, such as adaptive and learning ones, IST is always applied without correction of shaper's parameters. As a result, an issue of robustness against system uncertainties is highly valued [7]. In general, as the R-IST is the method based on traditional IST, damage of robustness is unwanted. For comparison of the robustness properties, the sensitivity curve is recalled. The relative amplitudes of residual vibration of the three methods when faced with uncertainty of system's natural frequency is tested. As shown in Figure 11, the robustness properties of these three methods are exhibited. It can be found that all these curves are different

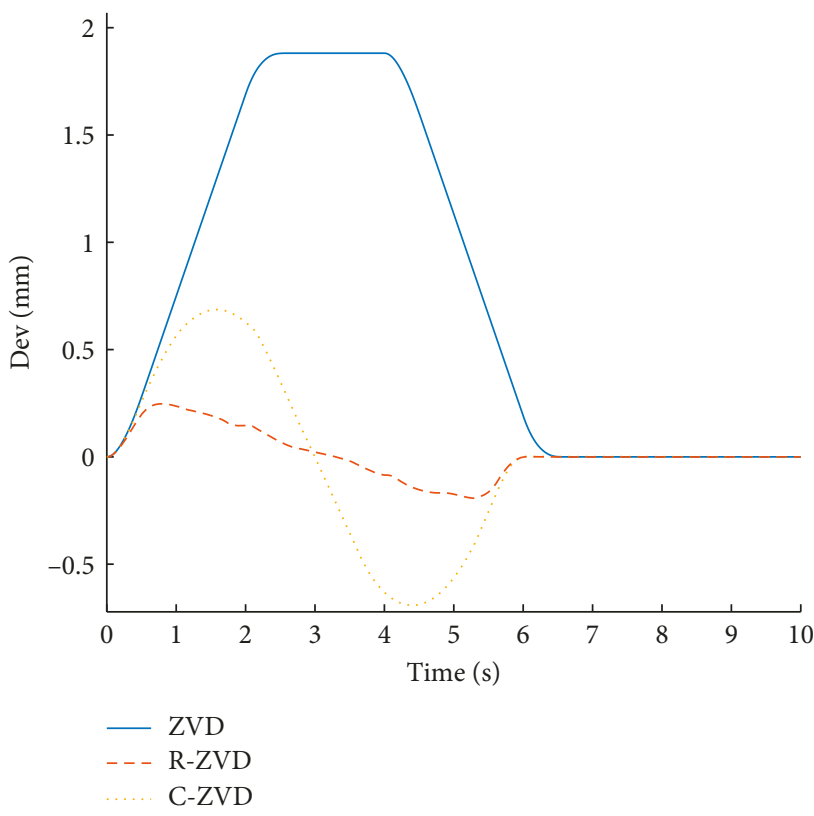

FIGURE 10: Trajectory deviations of mentioned methods.

with the ZVD robust curve obtained by impulse responses. In general, the robust curve of $\mathrm{ZVD}$, when tested through impulse responses, is closed to be symmetrical under $10 \%$ residual vibration [8]. This phenomenon is caused because the initial trajectory of ZVD and C-ZVD is trapezoidal, which has the ability to cancel residual vibration when choosing proper parameters. Although the robust curves of the three methods have the similar trend, the difference between them is also merit attention. Compared with ZVD and C-ZVD, R-ZVD method has different initial trajectory. ZVD and C-ZVD methods have not changed the initial trajectory, while R-ZVD has. The modification of the initial trajectory results in the loss of vibration cancellation of the initial trapezoidal trajectory. As for the similar trend of these three methods, similar trajectory should be the reason. In Figure 11, robust curve of C-ZVD and R-ZVD are homogeneous, and they have homogeneous trajectory, as shown in Figure 10. To be concluded, the robustness properties of the three methods are similar. Compared with $88.6 \%$ less trajectory deviation, the priority of C-ZVD is remarkable. However, for C-ZVD, the trajectory compression process will change the spectrum of initial trajectory, which may lead to unpredictable damage of the robustness. Although $\mathrm{R}-\mathrm{ZVD}$ also changes the spectrum, the additional filtering process highly alleviates the unpredictability, which gives the possibility of industrial application.

4.3. Trajectory Reversion Property. In Section 3, it should be noted that after trajectory reversion process, the obtained trajectory spectrum is more regular than the initial trajectory. This property is important in analyzing robustness. When R-ZVD is used, the frequency of stimulation is focused on $m \omega_{n}(m=1,3,5, \ldots)$, as shown in Figure 5(a). When R-IST based on ZV and ZVDD shaper is used, the frequency of stimulation is also focused on these frequencies, 


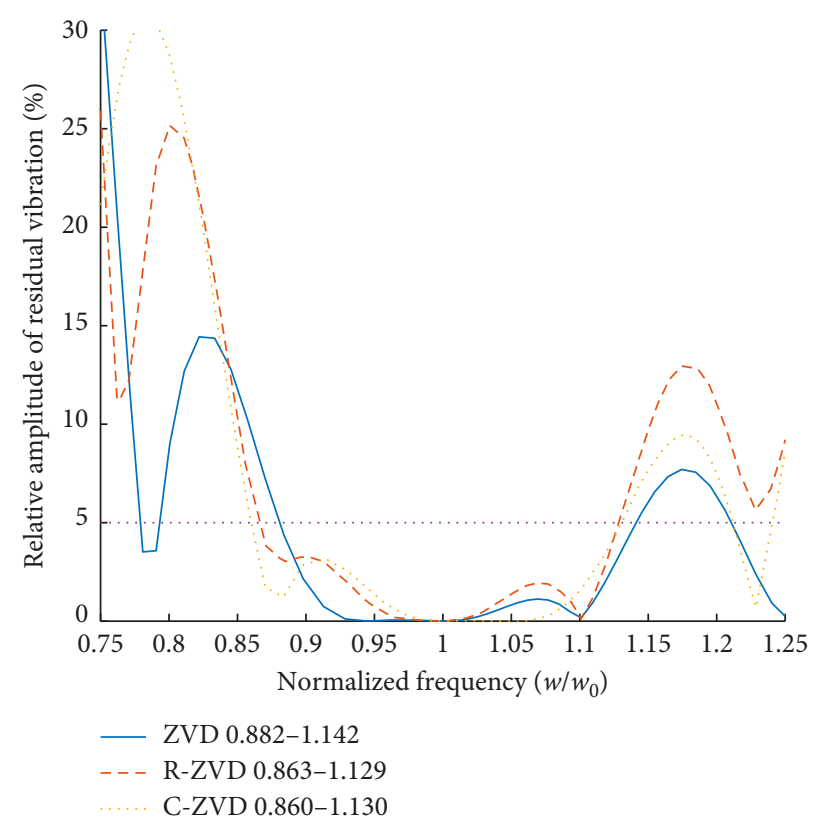

FIGURE 11: Robustness to uncertainty of nature frequency of different methods.

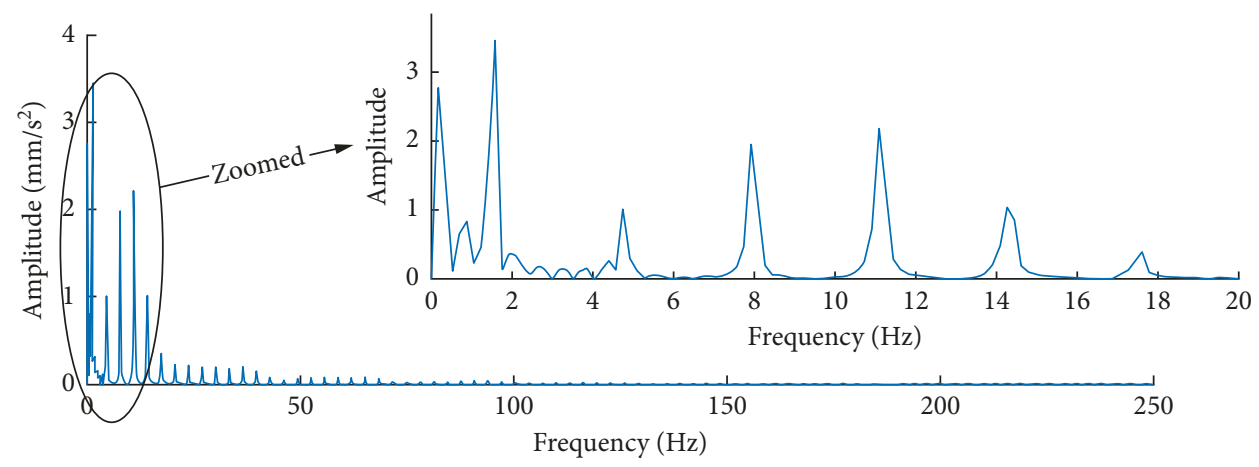

(a)

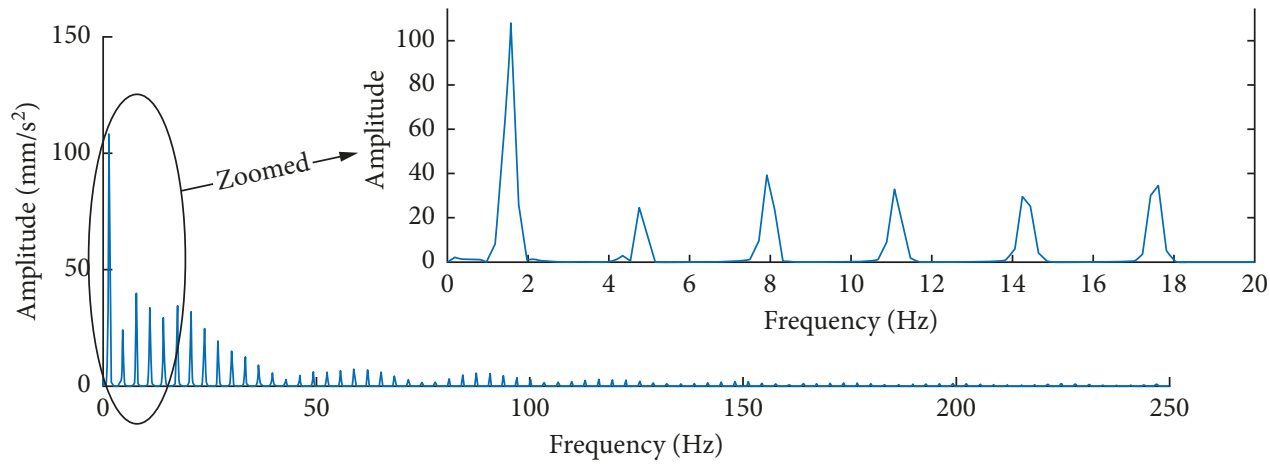

(b)

FIGURE 12: Acceleration spectrum obtained in reversion process using different principles: (a) ZV principle and (b) ZVDD principle.

as shown in Figure 12. To be detailed, the stimulating part on the frequency of $\omega_{n}$ is of the most remarkable. ZVDD method has the most concentrated frequency distributions. This phenomenon can be explained by two points:

First, LMS is aimed at reducing global trajectory deviation. In general, the target trajectory is continuous and smooth. Besides, the input shapers are a series of impulses, which means the shapers are discrete. The meaning of the reversion process is to generate a similar trajectory using the shapers. So, on both ends, the obtained trajectory is produced using not all of the impulses. To reduce the residual errors on the ends, extra displacements are added. Those 
displacements, which follow the cycle of odd times of the impulse interval, are strengthened. It is because after processed by the shapers, the displacements can be cancelled. As the frequency becomes higher, residual errors become smaller, and the effectiveness in reducing residual errors becomes less. However, another part of the displacements, which follow the cycle of even multiple numbers of the impulse interval, is suppressed. It is because these displacements will be enhanced in the overlapping area, which will enlarge residual errors. This reason can help explain why we chose the trajectory obtained by larger time interval as the target trajectory.

Second, the robustness of different shapers lead to different amplitudes of the frequency. The trajectory obtained directly from LMS leads to no residual vibration when the system parameter is already known. As the system parameter changed, residual vibration caused by residual error obtained by LMS becomes smaller. However, the vibration amplitude caused by target trajectory will also be changed. Through the principle of linear superposition, trajectory obtained in reversion process need to compensate the changes of vibration amplitude. However, the used shapers have certain robustness against the parameters' alteration. As a result, resonant components are brought in. These resonant components guarantee the conservation of vibration amplitude.

Finally, two important properties of the R-ZVD method can be obtained as follows.

Property 1: by utilizing the decomposition process based on different shapers, a new trajectory can be obtained with its excitation frequencies on specific value. In general, the period excitation parts are odd times of the impulse interval of chosen shaper.

Property 2: the amplitude on resonant frequency is decided by the robustness of chosen shaper. In general, the more robustness the shaper is, the bigger the amplitude of resonant part will be.

By using these two properties, proper input shaper can be selected according to the robust requests, and proper parameters of the filters to be used can be selected easily.

\section{Conclusions}

In this paper, a new trajectory optimization process is proposed, the so-called reversion-input-shaping technique (R-IST). A reversion process is added based on inputshaping principles. By using this process, stimulating frequency of origin trajectory is redistributed and focused on the specific frequency region, while leading to little alteration on other frequency components. This result leads to easy selection of resonance components to be wiped out. Furthermore, the reason of this decomposing phenomenon is discussed. Through simulation and analysis using ZVD shaper, the advantages and drawbacks of proposed R-IST were demonstrated. Although little damage of robustness properties could be caused by R-IST, the reduction of trajectory deviation is remarkable. In the future, research will be concentrated on three points. First, improve the R-IST to expand its application to time-varying systems. Second, combine R-IST with adaptive or learning IST. Third, combine R-IST with other kinds of input shapers.

\section{Data Availability}

The data used to support the findings of this study are available from the corresponding author upon request.

\section{Conflicts of Interest}

The authors declare no conflicts of interest.

\section{Acknowledgments}

This research was supported by the National Natural Science Foundation of China (Project no. 51475177) and State Key Lab of Digital Manufacturing Equipment \& Technology, School of Mechanical Science and Engineering, Huazhong University of Science \& Technology.

\section{References}

[1] D. Blackburn, W. Singhose, J. Kitchen et al., "Command shaping for nonlinear crane dynamics," Journal of Vibration and Control, vol. 16, no. 4, pp. 477-501, 2010.

[2] A. K. Banerjee and W. E. Singhose, "Command shaping in tracking control of a two-link flexible robot," Journal of Guidance, Control, and Dynamics, vol. 21, no. 6, pp. 10121015, 1998.

[3] K.-H. Rew and K.-S. Kim, "A closed-form solution to asymmetric motion profile allowing acceleration manipulation," IEEE Transactions on Industrial Electronics, vol. 57, no. 7, pp. 2499-2506, 2010.

[4] M. H. Korayem, A. Nikoobin, and V. Azimirad, "Trajectory optimization of flexible link manipulators in point-to-point motion," Robotica, vol. 27, no. 6, pp. 825-840, 2009.

[5] R. Kumar and T. Singh, "Design of input shapers using modal cost for multi-mode systems," Automatica, vol. 46, no. 3, pp. 598-604, 2010.

[6] W. Singhose, "Command shaping for flexible systems: a review of the first 50 years," International Journal of Precision Engineering and Manufacturing, vol. 10, no. 4, pp. 153-168, 2009.

[7] K.-H. Rew, C.-W. Ha, and K.-S. Kim, "An impulse-time perturbation approach for enhancing the robustness of extra-insensitive input shapers," Automatica, vol. 49, no. 11, pp. 3425-3431, 2013.

[8] N. C. Singer and W. P. Seering, "Preshaping command inputs to reduce system vibration," Journal of Dynamic Systems, Measurement, and Control, vol. 112, no. 1, pp. 76-82, 1990.

[9] W. Singhose, W. Seering, and N. Singer, "Residual vibration reduction using vector diagrams to generate shaped inputs," Journal of Mechanical Design, vol. 116, no. 2, pp. 654-659, 1994.

[10] U.-H. Park, J.-W. Lee, B.-D. Lim, and Y.-G. Sung, "Design and sensitivity analysis of an input shaping filter in the Z-plane," Journal of Sound and Vibration, vol. 243, no. 1, pp. 157-171, 2001.

[11] C. F. Cutforth and L. Y. Pao, "Adaptive input shaping for maneuvering flexible structures," Automatica, vol. 40, no. 4, pp. 685-693, 2004.

[12] P. H. Chang and H.-S. Park, "Time-varying input shaping technique applied to vibration reduction of an industrial 
robot," Control Engineering Practice, vol. 13, no. 1, pp. 121130, 2005.

[13] A. P. Tzes and S. Yurkovich, "Adaptive precompensators for flexible-link manipulator control," in Proceedings of the 28th IEEE Conference on Decision and Control, Tamba, FL, USA, December 1989.

[14] H. Yavuz, S. Mistıkoğlu, and S. Kapucu, "Hybrid input shaping to suppress residual vibration of flexible systems," Journal of Vibration and Control, vol. 18, no. 1, pp. 132-140, 2012.

[15] I. Duleba and J. Z. Sasiadek, "Nonholonomic motion planning based on newton algorithm with energy optimization," IEEE Transactions on Control Systems Technology, vol. 11, no. 3, pp. 355-363, 2003.

[16] Y. Fei, D. Fuqiang, and Z. Xifang, "Collision-free motion planning of dual-arm reconfigurable robots," Robotics and Computer-Integrated Manufacturing, vol. 20, no. 4, pp. 351357, 2004.

[17] J. Shan, H.-T. Liu, and D. Sun, "Modified input shaping for a rotating single-link flexible manipulator," Journal of Sound and Vibration, vol. 285, no. 1-2, pp. 187-207, 2005.

[18] K. L. Sorensen and W. E. Singhose, "Command-induced vibration analysis using input shaping principles," Automatica, vol. 44, no. 9, pp. 2392-2397, 2008.

[19] N. E. Huang, Z. Shen, S. R. Long et al., "The empirical mode decomposition and the hilbert spectrum for nonlinear and non-stationary time series analysis," Proceedings of the Royal Society of London A: Mathematical, Physical and Engineering Sciences, vol. 454, no. 1971, pp. 903-995, 1998.

[20] F. Ehrentreich and L. Sümmchen, "Spike removal and denoising of Raman spectra by wavelet transform methods," Analytical Chemistry, vol. 73, no. 17, pp. 4364-4373, 2001. 


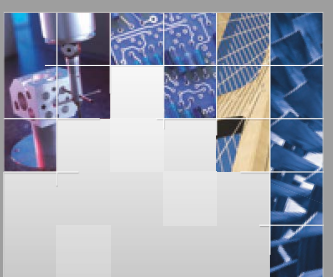

\section{Enfincering}
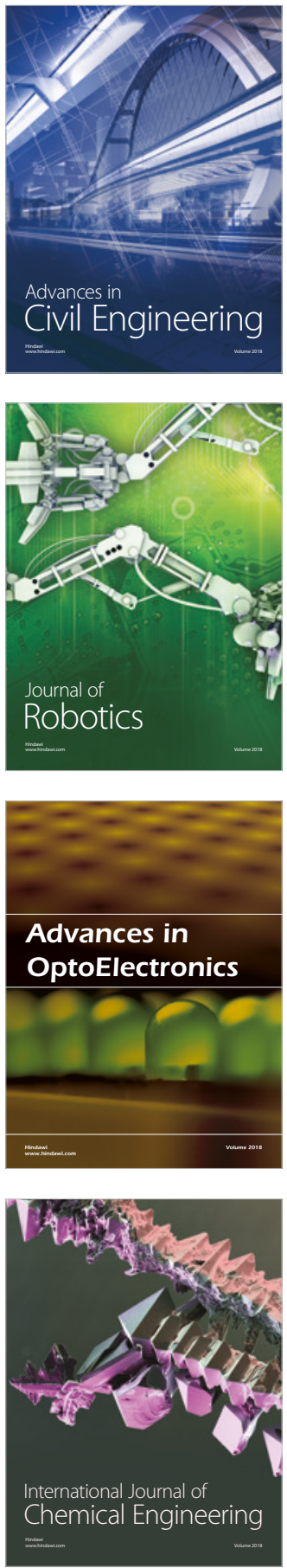

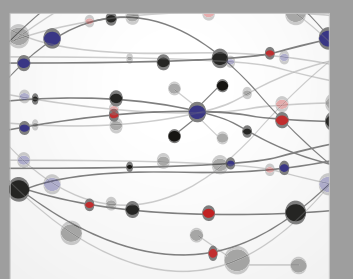

\section{Rotating \\ Machinery}

The Scientific World Journal

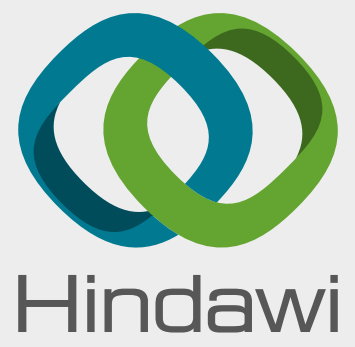

Submit your manuscripts at

www.hindawi.com
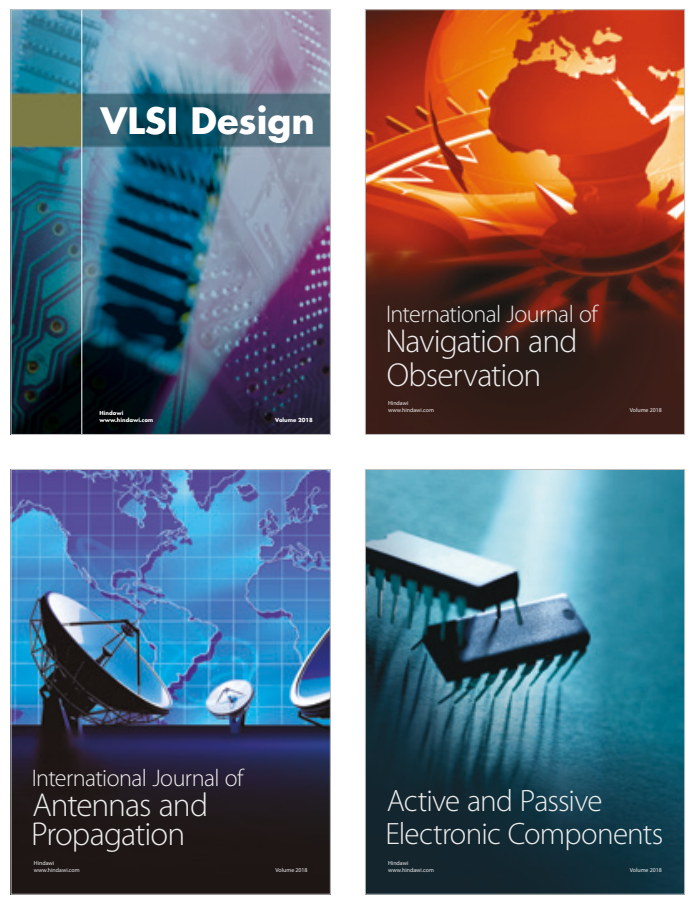
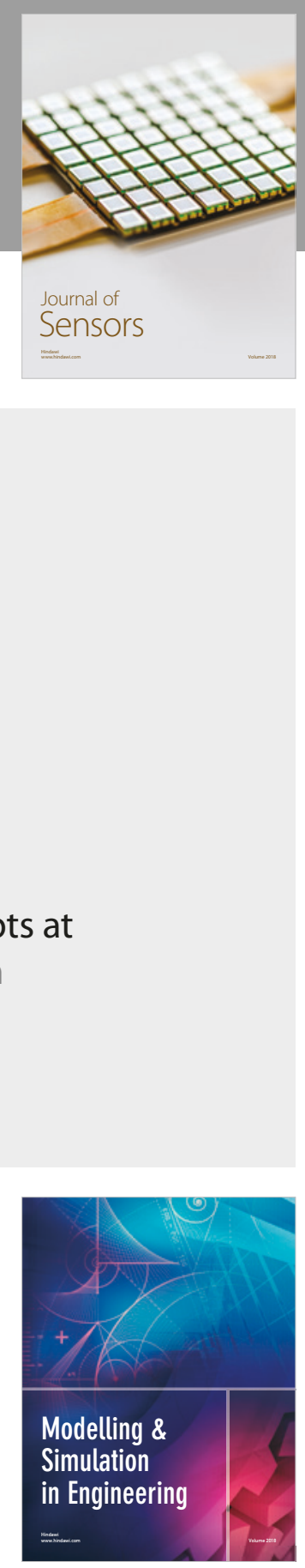

\section{Advances \\ Multimedia}
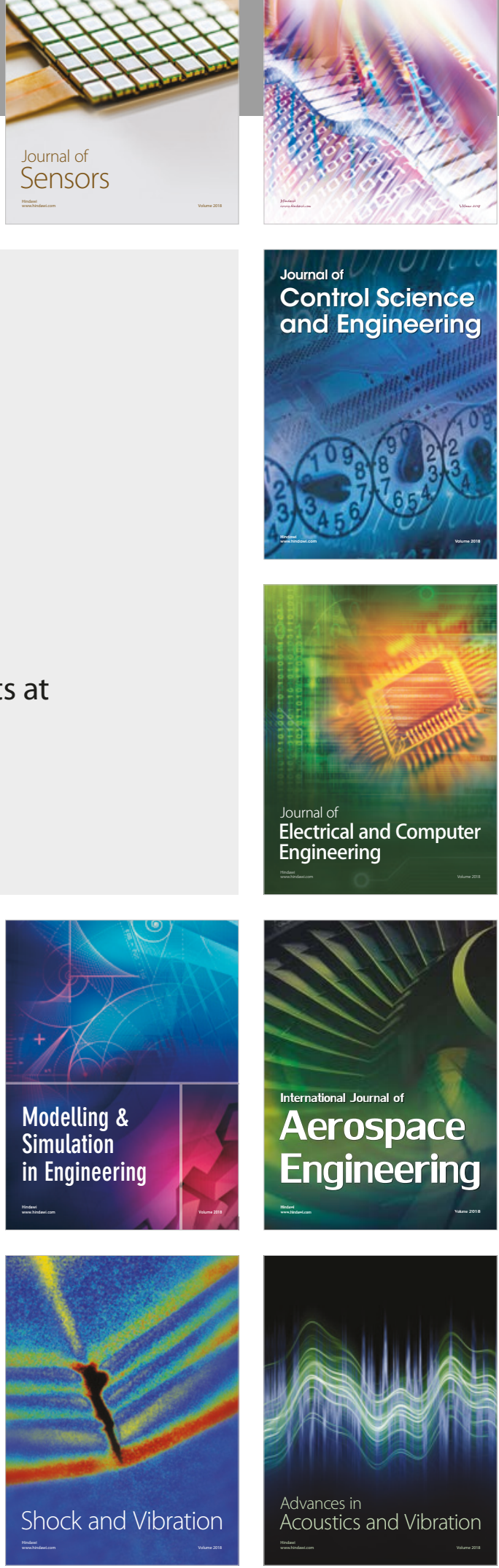